\title{
A Full List of Projectively Distinct K-Arcs in Finite Projetive Space Pg(2,8)
}

\section{Ali Ahmed A. Abdulla}

Abdulkhalik L. Yasin

ali2005aha@gmail.com

College of Computer Science and Mathematics,

University of Mosul

\section{Received on: 6/6/2011}

\section{ABSTRACT}

Accepted on: 16/8/2011

A $\mathrm{k}$-arc in a plane $\mathrm{PG}(2, \mathrm{q})$ is a set of $\mathrm{k}$ point such that every line in the plane intersect it in at most two points and there is a line intersect it in exactly two points. A $\mathrm{k}$-arc is complete if there is no $\mathrm{k}+1$-arc containing it. This thesis is concerned with studies a $\mathrm{k}$-arcs, $\mathrm{k}=4,5, \ldots, 10$ and classification of projectively distinct $\mathrm{k}$-arcs and distinct arcs under collineation. We prove by using computer program that the only complete $\mathrm{k}$-arcs is for, $\mathrm{k}=6,10$. This work take (150) hours computer time .

Keywords: projective space; complete arcs; Companion matrix

\section{القائمة الكاملة للأقواس-k المختلفة إسقاطيا في المستوي الإسقاطي (2,8)}

$$
\text { كلية علوم الحاسوب والرياضيات }
$$

جامعة الموصل

تاريخ قبول البحث: 2011/08/16

تاريخ استلام البحث: 2011/06/06

الملخص

القوس-k في المستوي PG(2,q) هو مجموعة من النقاط بحيث إن كل خط في المستوي يقطعه بما لا

يزيد عن نقطتين ويوجد خط يقطعه بنقطتين بالضبط، ويسمى القوس-k بأنه تام إذا لم يكن بالإمكان وجود قوس-

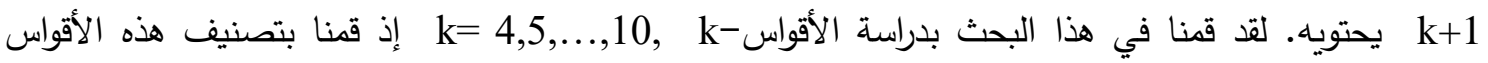

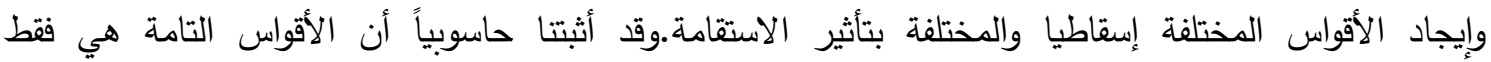
الأقواس-k=6,10,k. وقد استغرق منا العمل (150) ساعة حاسوبية. الكلمات المفتاحية: المستوى الاسقاطي ; القوس التام ; المصفوفة الإسقاطية.

يسمى القوس -k بأنه تام إذا لم يكن بالإمكان وجود قوس-k+1 يحتويه، لقد قام العالم [6 [6 مirschfeid بمجموعة من الدراسات للأقواس-k في المستوي الإسقاطي PG(2,q المعرفة على حقل كالوا GF(q) لقيم 99.

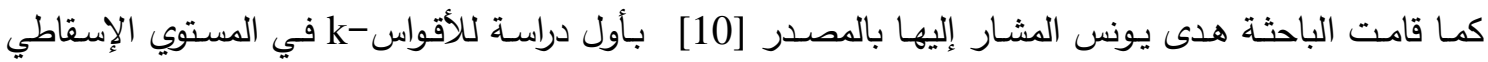

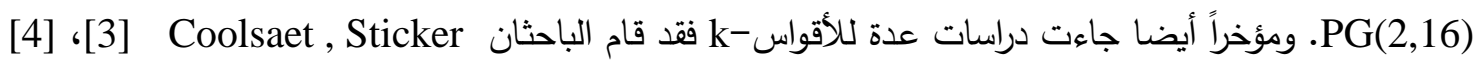

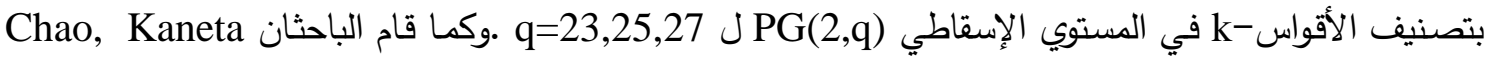

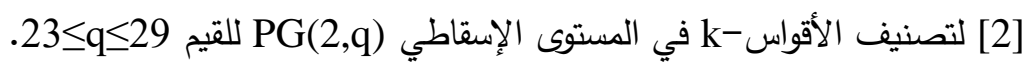
ومن الجدير بالذكر أن دراسة مثل هذا الموضوع المتتاول في هذا البحث يحتاج إلى فترة زمنية ليست بقصيرة لتتفيذ البرامج الحاسوبية التي استخدمت لإيجاد الأقواس-k المختلفة إسقاطية والأقواس -k المختلفة بتأثير الاستقامة. مثال : لتكن F(x)=x 


$$
\mathrm{GF}(8)=\left\{\sum_{i=0}^{h-1} a_{i} w^{i} / a_{i} \in Z_{2}, w^{3}+w+1=0\right\}, \mathrm{h}=3
$$

عناصر GF(8) هي

\begin{tabular}{|c|c|c|c|}
\hline$a_{0}$ & $a_{1}$ & $a_{2}$ & $a_{0}+a_{1} w^{+} a_{2} w^{2}$ \\
\hline 0 & 0 & 0 & 0 \\
\hline 0 & 1 & 0 & $w$ \\
\hline 0 & 1 & 1 & $w^{+} w^{2}$ \\
\hline 0 & 0 & 1 & $w^{2}$ \\
\hline 1 & 0 & 0 & 1 \\
\hline 1 & 1 & 0 & ${ }^{1+} w$ \\
\hline 1 & 0 & 1 & ${ }^{1+} w^{2}$ \\
\hline 1 & 1 & 1 & ${ }^{1+} w^{+} w^{2}$ \\
\hline
\end{tabular}

إن عناصر GF(8) هي:-

$\mathrm{GF}(8)=\left\{0,1, w, w^{2}, w^{3}, w^{4}, w^{5}, w^{6}: w^{7}=1\right\}, \quad$ هو جذر أولي $\quad$ ه هي:

[6] [6 [6 (1-2)

C(F) متعددة حدود أحادية، فإن المصفوفة المرافقة $F(x)=x^{n}-a n-1 x^{n-1}-\ldots$ - $a_{0}$ إذانة كانت

.n x n هي مصفوفة ذات بعد (Companion matrix)

$\mathrm{C}(\mathrm{F})=\left[\begin{array}{cccccc}0 & 1 & 0 & 0 & \ldots \ldots & 0 \\ 0 & 0 & 1 & 0 & \ldots . . & 0 \\ . & . & . & . & \ldots . & . \\ 0 & 0 & 0 & 0 & \ldots . & 1 \\ \mathrm{a}_{0} & \mathrm{a}_{1} & \mathrm{a}_{2} & \mathrm{a}_{3} & \ldots \ldots & \mathrm{a}_{\mathrm{n}-1}\end{array}\right]$

[6] [1-3) تعريف

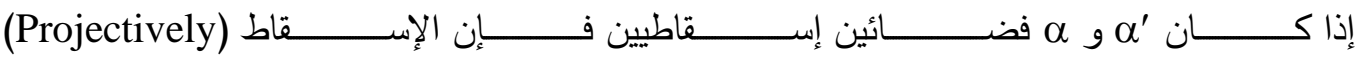

P(x') = P(x) S بحيث إذا كانت T تمثل بالمصفوفة غير المفردة (Bijection) هي علاقة تقابل الإن

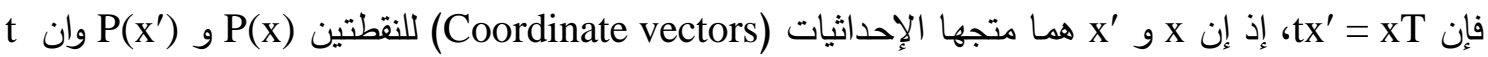
. $\in \mathrm{K}_{\mathrm{o}}$

(Principle of Duality) مبدأ الثنوية [6] (1-4)

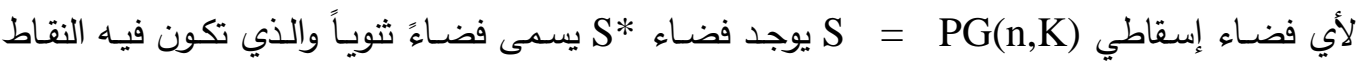

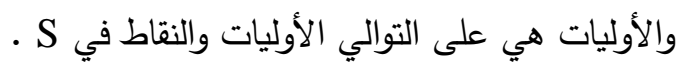


(6) [6 المبرهنة الأساسية في الهندسة الإسقاطية

\section{(Fundamental Theorem of Projective Geometry)}

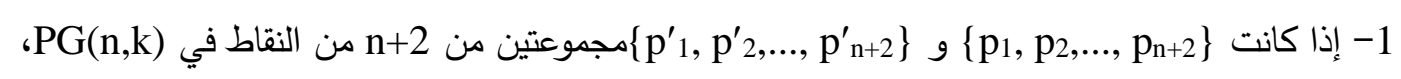

بحيث لا يوجد n+1 من النقاط المختارة من نفس المجموعة واقعة في أولي، فإن هناك إسقاطاً وحيداً S إذ

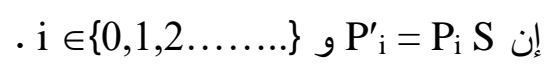

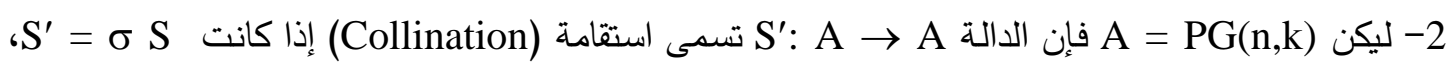

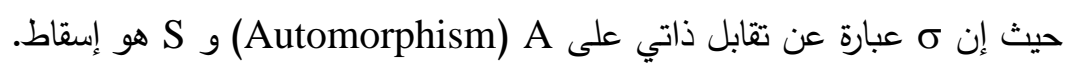

(1-6) (1-6) (الإسقاط الدوار (Cyclic Projectively)

ليكن T إسقاطاً في المستوي PG(n,q) فان T تسمى إسقاطاً دواراً إذا أمكن ترتيب نقاط الفضـاء كلها

$$
\mathrm{T}=\left[\begin{array}{ccc}
0 & 1 & 0 \\
0 & 0 & 1 \\
1 & 0 & \lambda^{3}
\end{array}\right]
$$

تترتب في دارة واحدة فقط. مثال : الإسقاط الآتي

حيث أن $\lambda$ هو جذر للحقل GF(8) إسقاط دوار. [6] [عريف

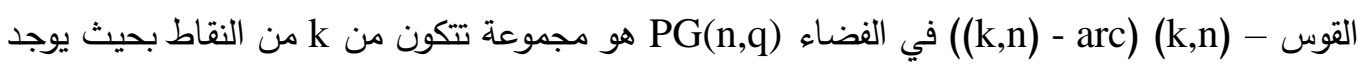

على الأكثر n منها على خط .ويوجد n منها بالضبط على الخط. عندما يكون n=2 فإن القوس - (k,2) يرمز لـ من له

$$
\text { بالقوس- }
$$

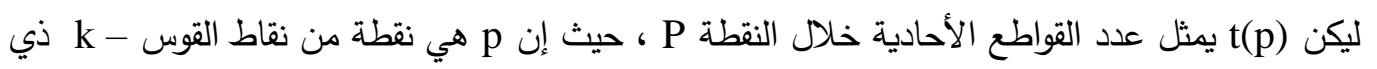

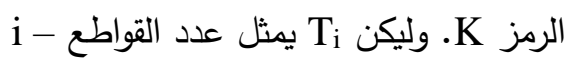

$$
\begin{aligned}
& \text { للقوس - k في المستوي فان : }
\end{aligned}
$$

$1-\mathrm{t}(\mathrm{p})=\mathrm{q}+2-\mathrm{k}=\mathrm{t}$

2- a. $\mathrm{T}_{2}=\mathrm{k}(\mathrm{k}-1) / 2$

b. $\mathrm{T}_{1}=\mathrm{kt}$

c. $\mathrm{T}_{\mathrm{o}}=\mathrm{q}(\mathrm{q}-1) / 2+\mathrm{t}(\mathrm{t}-1) / 2$

[6] (1-9)

يقال للقوس--(k,n) إنه تام (k+1,n) - إذا لم يكن هنالك قوس (complete) يحتويه.

[5] (1-10)

ألتربيعي (Quadric) هو أولوية ذات الرتبة 2 في الفضاء PG(n,q) كذلك إذا كانت R = V(F) حيث

إن F F ميغة تربيعية فأن : 
$\mathrm{F}=\sum_{i \leq j} a_{i j} x_{i} x_{j}=a_{00} x_{0}^{2}+a_{01} x_{0} x_{1}+a_{02} x_{0} x_{2}{ }^{+} a_{11} x_{1}^{2}+a_{12} x_{1} x_{2}{ }^{+} a_{22} x_{2}^{2}$

[6] (1-11)

التربيعي غير المفرد في المستوي (Conic) PG(2,q) يسمى مخروطي إن الصيغة العامة للمخروطي هي

PG(2,8) بناء وتصنيف الأقواس -k في المستوي الإسقاطي

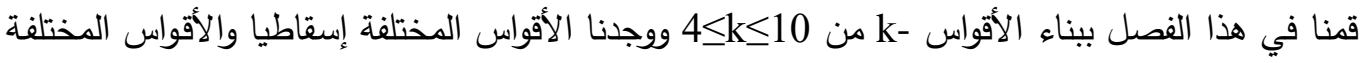
بتأثير الاستقامة كما وجدنا تأثير الزمرة PGL(3,8) على كل قوس من هذه الأقواس.

(PG(2,8) Plane) PG(2,8) المستوي (2-2)

المستوي PG(2,8 يحتوي على 73 نقطة و73 خطاً، وكل خط يحتوي على تسع نقاط وكل نقطة تقع

على تسعة خطوط.

ليكن GF(8) هو حقل كالوا للرتبة (8) فان عناصر هذا الحقل هي

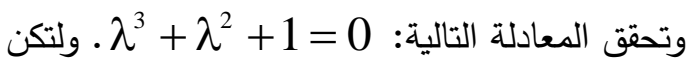

$C(f)=\left[\begin{array}{lll}0 & 1 & 0 \\ 0 & 0 & 1 \\ 1 & 0 & \lambda^{3}\end{array}\right]$

هي مصفوفة الوقوع التي تدور النقاط الـ 73 بدورة واحدة. ولتكن Po

$P_{i}=P_{i-1}\left[\begin{array}{lll}0 & 1 & 0 \\ 0 & 0 & 1 \\ 1 & 0 & \lambda^{3}\end{array}\right]^{i}, i=1,2, \ldots, 72$

إن النقاط الـ 73 التي تظهر موضحة في الجدول رقم (1):

جدول رقم (1). نقاط المستوي PG(2,8)

\begin{tabular}{|c|c|c|c|c|c|}
\hline I & points & I & points & I & points \\
\hline 0. & $(1,0,0)$ & 25. & $(1,1, \lambda)$ & 50. & $\left(1, \lambda, \lambda^{4}\right)$ \\
\hline 1. & $(0,1,0)$ & 26. & $\left(1, \lambda^{6}, \lambda^{5}\right)$ & 51. & $\left(1, \lambda^{3}, \lambda\right)$ \\
\hline 2. & $(0,0,1)$ & 27. & $\left(1, \lambda^{2}, \lambda^{4}\right)$ & 52. & $\left(1, \lambda^{6}, 1\right)$ \\
\hline 3. & $\left(1,0, \lambda^{3}\right)$ & 28. & $\left(1, \lambda^{3}, \lambda^{6}\right)$ & 53. & $\left(1,1, \lambda^{5}\right)$ \\
\hline 4. & $\left(1, \lambda^{4}, \lambda^{3}\right)$ & 29. & $(1, \lambda, \lambda)$ & 54. & $\left(1, \lambda^{2}, 1\right)$ \\
\hline 5. & $\left(1, \lambda^{4}, \lambda^{4}\right)$ & 30. & $\left(1, \lambda^{6}, \lambda^{2}\right)$ & 55. & $(1,1,1)$ \\
\hline 6. & $\left(1, \lambda^{3}, \lambda^{2}\right)$ & 31. & $\left(1, \lambda^{5}, \lambda\right)$ & 56. & $\left(1,1, \lambda^{2}\right)$ \\
\hline 7. & $\left(1, \lambda^{5}, \lambda^{4}\right)$ & 32. & $\left(1, \lambda^{6}, \lambda\right)$ & 57. & $\left(1, \lambda^{5}, \lambda^{6}\right)$ \\
\hline 8. & $\left(1, \lambda^{3}, \lambda^{4}\right)$ & 33. & $\left(1, \lambda^{6}, \lambda^{6}\right)$ & 58. & $\left(1, \lambda, \lambda^{5}\right)$ \\
\hline 9. & $\left(1, \lambda^{3}, \lambda^{5}\right)$ & 34. & $\left(1, \lambda, \lambda^{2}\right)$ & 59. & $\left(1, \lambda^{2}, 0\right)$ \\
\hline 10. & $\left(1, \lambda^{2}, \lambda^{6}\right)$ & 35. & $\left(1, \lambda^{5}, \lambda^{5}\right)$ & 60. & $\left(0,1, \lambda^{2}\right)$ \\
\hline 11. & $(1, \lambda, 0)$ & 36. & $\left(1, \lambda^{2}, \lambda^{2}\right)$ & 61. & $\left(1,0, \lambda^{6}\right)$ \\
\hline 12. & $(0,1, \lambda)$ & 37. & $\left(1, \lambda^{5}, \lambda^{2}\right)$ & 62. & $\left(1, \lambda, \lambda^{3}\right)$ \\
\hline 13. & $\left(1,0, \lambda^{5}\right)$ & 38. & $\left(1, \lambda^{5}, 0\right)$ & 63. & $\left(1, \lambda^{4}, \lambda^{6}\right)$ \\
\hline
\end{tabular}




\begin{tabular}{|c|c|l|c|l|c|}
\hline 14. & $\left(1, \lambda^{2}, \lambda^{3}\right)$ & 39. & $\left(0,1, \lambda^{5}\right)$ & 64. & $\left(1, \lambda, \lambda^{6}\right)$ \\
\hline 15. & $\left(1, \lambda^{4}, \lambda^{5}\right)$ & 40. & $(1,0,1)$ & 65. & $(1, \lambda, 1)$ \\
\hline 16. & $\left(1, \lambda^{2}, \lambda^{5}\right)$ & 41. & $\left(1,1, \lambda^{3}\right)$ & 66. & $\left(1,1, \lambda^{4}\right)$ \\
\hline 17. & $\left(1, \lambda^{2}, \lambda\right)$ & 42. & $\left(1, \lambda^{4}, \lambda\right)$ & 67. & $\left(1, \lambda^{3}, 0\right)$ \\
\hline 18. & $\left(1, \lambda^{6}, \lambda^{4}\right)$ & 43. & $\left(1, \lambda^{6}, 0\right)$ & 68. & $\left(0,1, \lambda^{3}\right)$ \\
\hline 19. & $\left(1, \lambda^{3}, 1\right)$ & 44. & $\left(0,1, \lambda^{6}\right)$ & 69. & $(1,0, \lambda)$ \\
\hline 20. & $(1,1,0)$ & 45. & $\left(1,0, \lambda^{4}\right)$ & 70. & $\left(1, \lambda^{6}, \lambda^{3}\right)$ \\
\hline 21. & $(0,1,1)$ & 46. & $\left(1, \lambda^{3}, \lambda^{3}\right)$ & 71. & $\left(1, \lambda^{4}, 0\right)$ \\
\hline 22. & $\left(1,0, \lambda^{2}\right)$ & 47. & $\left(1, \lambda^{4}, \lambda^{2}\right)$ & 72. & $\left(0,1, \lambda^{4}\right)$ \\
\hline 23. & $\left(1, \lambda^{5}, \lambda^{3}\right)$ & 48. & $\left(1, \lambda^{5}, 1\right)$ & & \\
\hline 24. & $\left(1, \lambda^{4}, 1\right)$ & 49. & $\left(1,1, \lambda^{6}\right)$ & & \\
\hline
\end{tabular}

ليكن L هو خط في المستوي ونأخذ هو الخط في المالانهاية أي إن X $\begin{array}{lllllllll}0 & 1 & 11 & 20 & 38 & 43 & 59 & 67 & 71\end{array}$

بما إن المصفوفة المرافقة C(f تُدور جميع نقاط المستوي بدورة واحدة وبما إن النقاط والخطوط احدهما مثنى

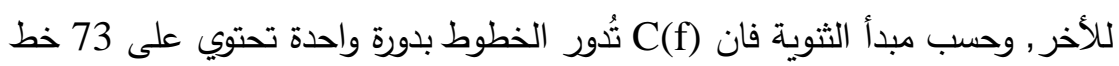

$\begin{array}{lllllllll}72 & 0 & 10 & 19 & 37 & 42 & 58 & 66 & 70\end{array}$ ويكون الخط الأخير

(Construction k-arcs for, k=4,5,...,10) k=4,5, ...,10, k-

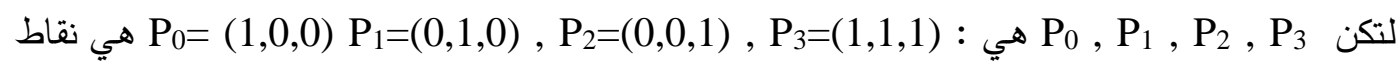
المصدر والتي تثكل القوس -4 وحسب المبرهنـة الأساسية في الإسـقاط (8-1) فـان القوس-4 يكافئ القوس وعليه فانه يمكن بناء الأقواس من k=5 إلى k=10 بالاعتماد على القوس-4 الذي يضم النقاط وحسب الخوارزمية التالية المستخدمة بالبرنامج الحاسوبي:-

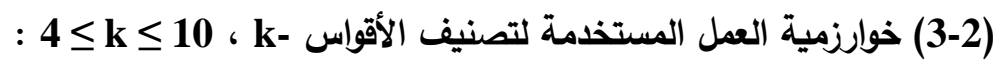
أولا: تعين الخطوط ذات القاطع -2 للقوس $\mathrm{K}=\left\{\mathrm{P}_{0}, \mathrm{P}_{1}, \mathrm{P}_{2}, \mathrm{P}_{3}\right\}$

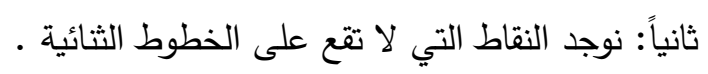

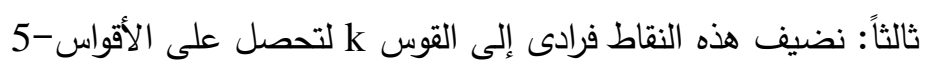

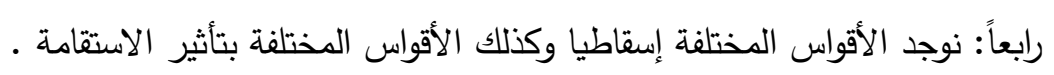
خامساً: نوجد الزمر التي تثبت هذه الأقواس التواس

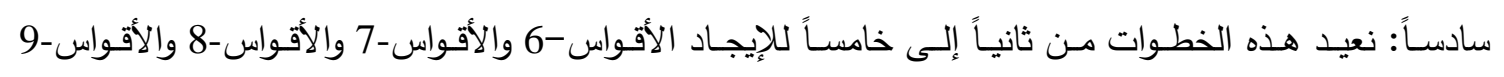

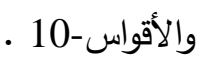
سابعاً: نوجد الأقواس التامة لجميع الخطوات إن وجدت . (3-3) الأقواس المختلفة إسقاطيا والمختلفة بتأثير الاستقامة (K-arc Projectively Distinct and Collnieation Distinct)

(3-3-1) الأقواس-5 المختلفة إسقاطيا والمختلفة بتأثير الاستقامة لبناء الأقواس-5 نؤشر نقاط المصدر الأربعة على خطوط المستوي ونحذف نقاط الخطوط ذات القاطع الثنائي من نقاط المستوي PG(2.8 ثم نضيف النقاط فِرادى إلى نقاط المصدر فنحصل على الأقواس-5. 
وباستخدام البرنامج الحاسوبي لإيجاد الأقواس-5 المختلفة إسقاطيا وجدنا إن عدد الأقواس المختلفة إسقاطيا هو $(1,0,0),(0,1,0),(0,0,1),(1,1,1),\left(1,, \lambda^{4}, \lambda^{3}\right)$ قوس واحد والذي نقاطه هي:إن زمرة هذا القوس هي CC $\emptyset_{1}:\left(\mathrm{X}_{0}, \mathrm{X}_{1}, \mathrm{X}_{2}\right) \rightarrow\left[\begin{array}{ccc}1 & 0 & 0 \\ 0 & 0 & \lambda^{4} \\ 0 & \lambda^{3} & 0\end{array}\right]\left[\begin{array}{c}X_{0} \\ X_{1} \\ X_{2}\end{array}\right]$ $\emptyset_{2}:\left(\mathrm{X}_{0}, \mathrm{X}_{1}, \mathrm{X}_{2}\right) \rightarrow\left[\begin{array}{ccc}1 & \lambda & \lambda^{5} \\ 0 & \lambda^{5} & \lambda^{5} \\ 0 & \lambda^{4} & \lambda^{5}\end{array}\right]\left[\begin{array}{l}X_{0} \\ X_{1} \\ X_{2}\end{array}\right]$ التحويل Ø المدار الأول $\{(0,1,0),(0,0,1)$ والمدار الثاني $\left\{(1,1,1),\left(1, \lambda^{4}, \lambda^{3}\right)\right\}$ التحويل والمدار الأول $\}$ والمدار الثاني $\{(0,0,1),(1,1,1)$ القوس-5 محتوى في المخروطي : $\mathrm{V}\left(X_{0} X_{1}+\lambda^{4} X_{0} X_{2}+\lambda^{6} X_{1} X_{2}\right)$

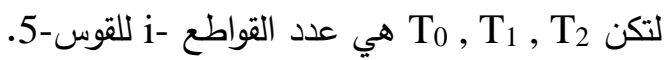

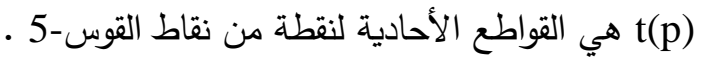
حسب المبرهنة (12-1) فان $\mathrm{t}(\mathrm{p})=5, \mathrm{~T}_{0}=38, \mathrm{~T}_{1}=25,, \mathrm{~T}_{2}=10$

بما إن عدد الأقواس-5 المختلفة إسقاطيا هو واحد. فان عدد الأقواس-5 المختلفة بتأثير الاستقامة هي واحد أيضا. (3-3-2) الأقواس-6 المختلفة إسقاطيا والمختلفة بتأثير الاستقامة

بنفس الطريقة في (1-3-3) نحصل على خمسة أقواس-6 مختلفة إسقاطيا كما في الجدول رقم (2). جلول رقم (2). الأقواس-6 المختلفة إسقاطيا

\begin{tabular}{|l|l|l|l|l|l|l|l|l|}
\hline & $\mathrm{P}_{0}$ & $\mathrm{P}_{1}$ & $\mathrm{P}_{2}$ & $\mathrm{P} 3$ & $\mathrm{P}_{4}$ & $\mathrm{P}_{5}$ & $\mathrm{G}$ & $\mid \mathrm{G}$ \\
\hline $\mathrm{X}_{0}$ & 0 & 1 & 2 & 55 & 4 & 8 & $\mathrm{C}_{2} \times \mathrm{C}_{2}$ & 4 \\
$\mathrm{X}_{2}$ & 0 & 1 & 2 & 55 & 4 & 9 & $\mathrm{~S}_{3}$ & 6 \\
$\mathrm{X}_{3}$ & 0 & 1 & 2 & 55 & 4 & 28 & $\mathrm{~S}_{4}$ & 24 \\
$\mathrm{X}_{4}$ & 0 & 1 & 2 & 55 & 4 & 30 & $\mathrm{~S}_{4}$ & 24 \\
$\mathrm{X}_{5}$ & 0 & 1 & 2 & 55 & 4 & 50 & $\mathrm{~S}_{4}$ & 24 \\
\hline
\end{tabular}

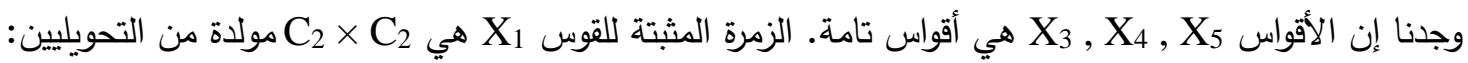




$$
\begin{aligned}
& \emptyset_{1}:\left(X_{0}, X_{1}, X_{2}\right) \rightarrow\left[\begin{array}{ccc}
1 & 0 & 0 \\
0 & 0 & 1 \\
0 & 1 & 0
\end{array}\right]\left[\begin{array}{l}
X_{0} \\
X_{1} \\
X_{2}
\end{array}\right] \\
& \emptyset_{2}:\left(\mathrm{X}_{0}, \mathrm{X}_{1}, \mathrm{X}_{2}\right) \rightarrow\left[\begin{array}{ccc}
1 & \lambda^{6} & \lambda^{6} \\
0 & \lambda^{3} & \lambda^{2} \\
0 & \lambda^{2} & \lambda^{3}
\end{array}\right]\left[\begin{array}{l}
X_{0} \\
X_{1} \\
X_{2}
\end{array}\right] \\
& \text { التحويل Ø يثبت النقاط (1,1,1) , (1,0,0) ويقسم باقي نقاط القوس إلى مدارين هما: } \\
& \text { المدار الأول : }\}
\end{aligned}
$$

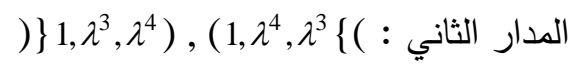

التحويل Ø يثبت النقاط (1,1,1) , (1,0,0) ويقسم باقي نقاط القوس إلى مدارين أيضا هما:

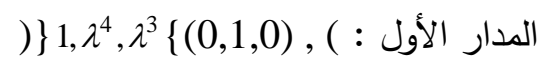
المدار الثاني : ( ) , ( $1, \lambda^{3}, \lambda^{4}\{(0,0,1)$ إن المخروطي (يحتوي على خمس نقاط من نقاط القوس X $X_{0} X_{1}+\lambda^{4} X_{0} X_{2}+\lambda^{6} X_{1} X_{2}$ والتي هي $\begin{array}{lllll}4 & 55 & 2 & 1 & 0\end{array}$

$\emptyset_{1}:\left(X_{0}, X_{1}, X_{2}\right) \rightarrow\left[\begin{array}{lll}0 & 1 & 1 \\ 0 & 1 & 0 \\ 1 & 1 & 0\end{array}\right]\left[\begin{array}{l}X_{0} \\ X_{1} \\ X_{2}\end{array}\right]$
$\emptyset_{2}:\left(X_{0}, X_{1}, X_{2}\right) \rightarrow\left[\begin{array}{ccc}1 & \lambda^{5} & 1 \\ \lambda^{3} & 0 & 1 \\ \lambda^{5} & 0 & 1\end{array}\right]\left[\begin{array}{l}X_{0} \\ X_{1} \\ X_{2}\end{array}\right]$

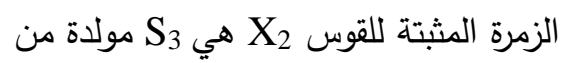

$$
\begin{aligned}
& \text { التحويل } \\
& \text { المدار الأول: }\} \\
& \text { الددار الثاني: }
\end{aligned}
$$

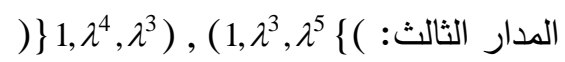

$$
\begin{aligned}
& \text { التحويل Ø }
\end{aligned}
$$

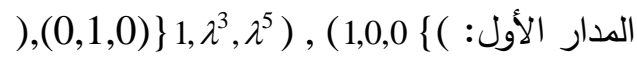

$$
\begin{aligned}
& \text { المدار الثاني: (0,0,1 (1,1,1) }
\end{aligned}
$$

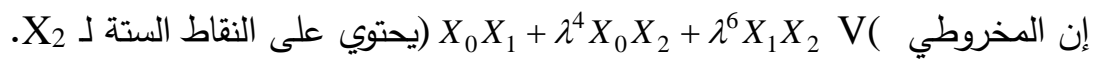
نلاحظ إن الزمرة المثبتة للأقواس X مخروطي واحد. لقد استخدمنا برنامجا collineation للإيجاد الأقواس المختلفة بتأثير الاستقامة فحصلنا على ثلاثة أقواس فقط كما هو موضع في الجدول التالي: 


جدول رقم (3). الأقواس -6 - المختلفة بتأثير الاستقامة
\begin{tabular}{|l|l|l|l|l|l|l|}
\hline & $\mathrm{P}_{0}$ & $\mathrm{P}_{1}$ & $\mathrm{P}_{2}$ & $\mathrm{P}_{3}$ & $\mathrm{P}_{4}$ & $\mathrm{P}_{5}$ \\
\hline $\mathrm{Y}_{1}$ & 0 & 1 & 2 & 55 & 4 & 8 \\
$\mathrm{Y}_{2}$ & 0 & 1 & 2 & 55 & 4 & 9 \\
$\mathrm{Y}_{3}$ & 0 & 1 & 2 & 55 & 4 & 28 \\
\hline
\end{tabular}

إن القوس Y3 هو قوس تام. (3-3-3) الأقواس-7 المختلفة إسقاطيا والمختلفة بتأثير الاستقامة بنفس الطريقة في (1-3-3) حصلنا على قوسين مختلفين إسقاطيا كما في الجدول التالي:جدول رقم (4). الأقواس-7 المختلفة إسقاطيا

\begin{tabular}{|l|l|l|l|l|l|l|l|l|l|}
\hline & $\mathrm{P}_{0}$ & $\mathrm{P}_{1}$ & $\mathrm{P}_{2}$ & $\mathrm{P}_{3}$ & $\mathrm{P}_{4}$ & $\mathrm{P}_{5}$ & $\mathrm{P}_{6}$ & $\mathrm{G}$ & $|\mathrm{G}|$ \\
\hline $\mathrm{Z}_{1}$ & 0 & 1 & 2 & 55 & 4 & 8 & 16 & $\mathrm{~S}_{3}$ & 6 \\
$\mathrm{Z}_{2}$ & 0 & 1 & 2 & 55 & 4 & 9 & 10 & $\mathrm{D}_{7}$ & 14 \\
\hline
\end{tabular}

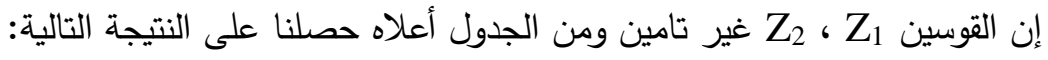
نتيجة: لا يوجد قوس -7 تام في المستوي (2,8) إن القوس Z

$$
\begin{aligned}
& \emptyset_{1}:\left(\mathrm{X}_{0}, \mathrm{X}_{1}, \mathrm{X}_{2}\right) \rightarrow\left[\begin{array}{ccc}
1 & \lambda^{5} & \lambda \\
0 & \lambda^{5} & \lambda^{4} \\
0 & \lambda^{5} & \lambda^{5}
\end{array}\right]\left[\begin{array}{l}
X_{0} \\
X_{1} \\
X_{2}
\end{array}\right] \\
& \emptyset_{2}:\left(\mathrm{X}_{0}, \mathrm{X}_{1}, \mathrm{X}_{2}\right) \rightarrow\left[\begin{array}{ccc}
1 & 0 & \lambda^{4} \\
0 & 0 & \lambda \\
0 & \lambda^{6} & 1
\end{array}\right]\left[\begin{array}{l}
X_{0} \\
X_{1} \\
X_{2}
\end{array}\right]
\end{aligned}
$$

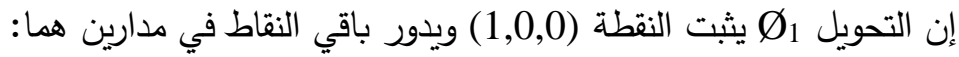

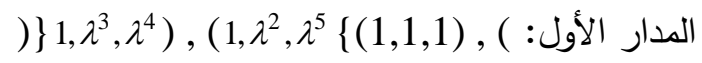

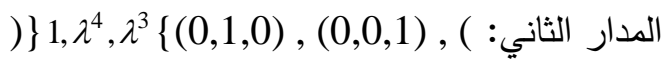

$$
\begin{aligned}
& \text { وان التحويل Ø يثبت النقطة }(1,0,0) \text { أيضا ويدور باقي نقاط القوس في ثلاثة مدارات هي: } \\
& \text { المدار الأول: ( ) } \left.1, \lambda^{2}, \lambda^{5}\right),\left(1, \lambda^{4}, \lambda^{3}\right.
\end{aligned}
$$

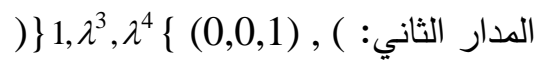

$$
\begin{aligned}
& \text { المدار الثالث: }\}
\end{aligned}
$$

المخروطي ( يحتوي على خمسة نقاط من Z $X_{0} X_{1}+\lambda^{4} X_{0} X_{2}+\lambda^{6} X_{1} X_{2} \mathrm{~V}$ ونقطنين خارجيتين. إن القوس Z يمتلك الزمرة D المولدة من التحويليين:

$$
\begin{aligned}
& \emptyset_{1}:\left(\mathrm{X}_{0}, \mathrm{X}_{1}, \mathrm{X}_{2}\right) \rightarrow\left[\begin{array}{ccc}
1 & 0 & 0 \\
0 & 0 & \lambda^{4} \\
0 & \lambda^{3} & 0
\end{array}\right]\left[\begin{array}{l}
X_{0} \\
X_{1} \\
X_{2}
\end{array}\right] \\
& \emptyset_{2}:\left(\mathrm{X}_{0}, \mathrm{X}_{1}, \mathrm{X}_{2}\right) \rightarrow\left[\begin{array}{ccc}
0 & \lambda^{6} & \lambda^{4} \\
\lambda^{4} & \lambda & 0 \\
0 & \lambda^{5} & 0
\end{array}\right]\left[\begin{array}{l}
X_{0} \\
X_{1} \\
X_{2}
\end{array}\right]
\end{aligned}
$$


ذي الرتب 2 , 7 على التوالي.

إن التحويل

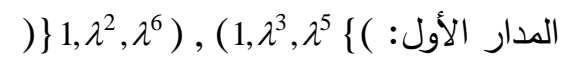

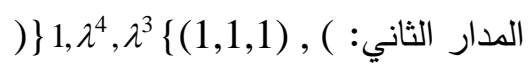

المدار الثالث: $\}$

وان التحويل Ø

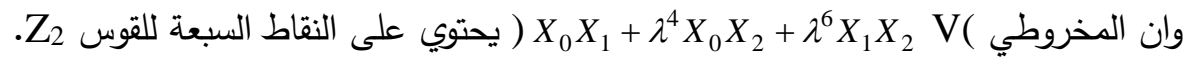
ولإيجاد الأقواس-7 المختلفين بتأثير الاستقامة استخدمنا البرنامج الحاسوبي Collineation فحصلنا على قوسين فقط وهما نفس القوسين Z Z

(3-3-4) الأقواس-8 المختلفة إسقاطيا والمختلفة بتأثير الاستقامة

بنفس الطريقة في (1-3-3) حصلنا على قوسين مختلفين إسقاطيا كما في الجدول التالي:جدول رقم (5). الأقواس-8 المختلفة إسقاطيا

\begin{tabular}{|l|l|l|l|l|l|l|l|l|c|c|}
\hline & $\mathrm{P}_{0}$ & $\mathrm{P}_{1}$ & $\mathrm{P}_{2}$ & $\mathrm{P}_{3}$ & $\mathrm{P}_{4}$ & $\mathrm{P}_{5}$ & $\mathrm{P}_{6}$ & $\mathrm{P}_{7}$ & $\mathrm{G}$ & $\mathrm{G}$ \\
\hline $\mathrm{F}_{1}$ & 0 & 1 & 2 & 55 & 4 & 8 & 16 & 32 & $\mathrm{D}_{4}$ & 14 \\
$\mathrm{~F}_{2}$ & 0 & 1 & 2 & 55 & 4 & 9 & 10 & 18 & $\mathrm{PGL}(2,16)$ & 56 \\
\hline
\end{tabular}

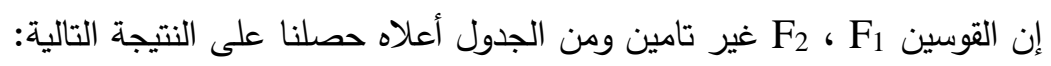
نتيجة: لا يوجد قوس-8 تام في المستوي PG) $\emptyset_{1}:\left(\mathrm{X}_{0}, \mathrm{X}_{1}, \mathrm{X}_{2}\right) \rightarrow\left[\begin{array}{ccc}1 & 0 & 0 \\ 0 & 0 & \lambda^{6} \\ 0 & \lambda & 0\end{array}\right]\left[\begin{array}{l}X_{0} \\ X_{1} \\ X_{2}\end{array}\right]$
$\emptyset_{2}:\left(\mathrm{X}_{0}, \mathrm{X}_{1}, \mathrm{X}_{2}\right) \rightarrow\left[\begin{array}{ccc}1 & 1 & \lambda^{5} \\ 0 & \lambda^{3} & \lambda^{2} \\ 0 & \lambda^{4} & 1\end{array}\right]\left[\begin{array}{l}X_{0} \\ X_{1} \\ X_{2}\end{array}\right]$ إن القوس F1 يمتلك الزمرة D D المولدة من التحويليين:

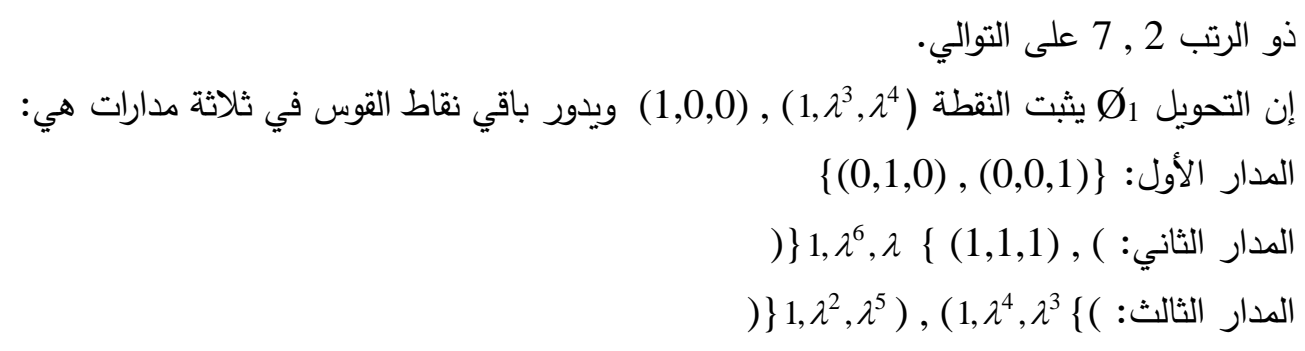
وان التحويل $\varnothing_{2}$ يثبت النقطة (1,0,0) أيضا ويُّور باقي نقاط القوس في مدار واحد هو:$\left\{(0,1,0),\left(1, \lambda^{3}, \lambda^{4}\right),(0,0,1),\left(1, \lambda^{4}, \lambda^{5}\right),\left(1, \lambda^{6}, \lambda\right),(1,1,1),\left(1, \lambda^{2}, \lambda^{5}\right)\right\}$

المخروطي ( يحتوي على خمسة نقاط من $X_{0} X_{1}+\lambda^{4} X_{0} X_{2}+\lambda^{6} X_{1} X_{2}$ و وثلاثة نقاط خارجية. إن القوس FG متكون من زمرة

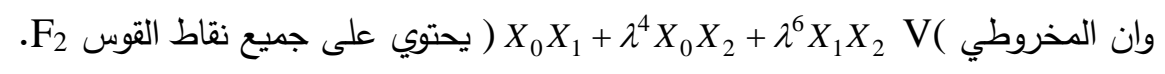


ولإيجاد الأقواس-8 المختلفين بتأثير الاستقامة استخدمنا البرنامج الحاسوبي Collineation فحصلنا على قوسين

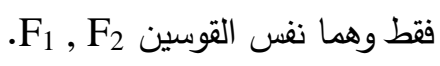

(3-3-5) الأقواس-9 المختلفة إسقاطيا والمختلفة بتأثير الاستقامة

بنفس الطريقة في (1-3-3) حصلنا على قوسين مختلفين إسقاطيا كما في الجدول التالي. جدول رقم (6). الأقواس -9 المختلفة إسقاطيا

\begin{tabular}{|l|l|l|l|l|l|l|l|l|l|l|l|}
\hline & $\mathrm{P}_{0}$ & $\mathrm{P}_{1}$ & $\mathrm{P}_{2}$ & $\mathrm{P}_{3}$ & $\mathrm{P}_{4}$ & $\mathrm{P}_{5}$ & $\mathrm{P}_{6}$ & $\mathrm{P}_{7}$ & $\mathrm{P}_{8}$ & $\mathrm{G}$ & $\mid \mathrm{G}$ \\
\hline $\mathrm{H}_{1}$ & 0 & 1 & 2 & 55 & 4 & 8 & 16 & 32 & 37 & $\operatorname{PGL}(2,16)$ & 56 \\
$\mathrm{H}_{2}$ & 0 & 1 & 2 & 55 & 4 & 9 & 10 & 18 & 34 & $\operatorname{PGO}(3,16)$ & 504 \\
\hline
\end{tabular}

إن القوسين H2 H نتيجة: لا يوجد قوس -9 تام في المستوي P(2.8)

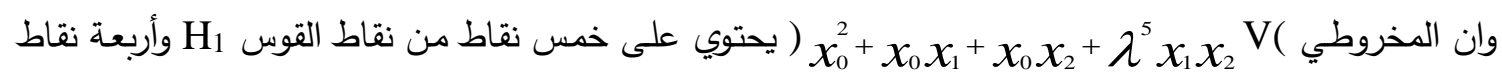
تقع خارجه. والمخروطي هئ ولإيجاد الأقواس -9 المختلفين بتأثير الاستقامة استخدمنا البرنامج الحاسوبي Collineation فحصلنا على قوسين فقط هما $\mathrm{H}_{1}, \mathrm{H}_{2}$ (3-3-6) الأقواس-10 المختلفة إسقاطيا والمختلفة بتأثير الاستقامة إن عدد الأقواس-9 المختلفة إسقاطيا هو قوسين فقط وهما He

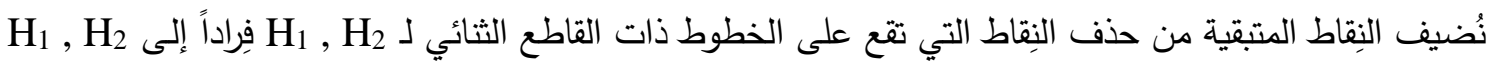
فنحصل على قوسين فقط. وباستخدام البرنامج الحاسوبي A حصلنا على قوس تام واحد كما في الجدول التالي جدول رقم (7). الأقواس -10 المختلفة إسقاطيا

\begin{tabular}{|l|l|l|l|l|l|l|l|l|l|l|l|l|}
\hline & $\mathrm{P}_{0}$ & $\mathrm{P}_{1}$ & $\mathrm{P}_{2}$ & $\mathrm{P}_{3}$ & $\mathrm{P}_{4}$ & $\mathrm{P}_{5}$ & $\mathrm{P}_{6}$ & $\mathrm{P}_{7}$ & $\mathrm{P}_{8}$ & $\mathrm{P}_{9}$ & $\mathrm{G}$ & $\mid \mathrm{G}$ \\
\hline $\mathrm{J}$ & 0 & 1 & 2 & 55 & 4 & 8 & 16 & 32 & 37 & 64 & $\operatorname{PGO}(3,16)$ & 504 \\
\hline
\end{tabular}

(The Results) (4) (4)

(4-2) الأقواس-k المختلفة إسقاطيا

الجدول رقم (3) يمثل التصنيف الكامل للأقواس المختلفة إسقاطيا (k=5,..10) إذ إن Nk يمثل عدد الأقواس المختلفة إسقاطيا. Gs يمثل زمرة القوس -k م . وقد استغرق من العمل الحاسوبي للحصول على هذه النتائج (150) ساعة حاسوبية

جدول رقم (8) - (8)

\begin{tabular}{|c|c|c|c|c|c|c|c|c|c|c|c|}
\hline \multicolumn{2}{|c|}{$\begin{array}{l}\mathrm{k}=5 \\
\mathrm{Nk}=1\end{array}$} & \multicolumn{2}{|l|}{$\begin{array}{l}\mathrm{k}=6 \\
\mathrm{Nk}=5\end{array}$} & \multicolumn{2}{|c|}{$\begin{array}{l}\mathrm{k}=7 \\
\mathrm{Nk}=2\end{array}$} & \multicolumn{2}{|l|}{$\begin{array}{l}\mathrm{k}=8 \\
\mathrm{Nk}=2\end{array}$} & \multicolumn{2}{|l|}{$\begin{array}{l}\mathrm{k}=9 \\
\mathrm{Nk}=2\end{array}$} & \multicolumn{2}{|l|}{$\begin{array}{l}\mathrm{k}=10 \\
\mathrm{Nk}=1\end{array}$} \\
\hline Gs & \# & Gs & \# & Gs & $\#$ & Gs & \# & Gs & \# & Gs & \# \\
\hline $\mathrm{C}_{2} \times \mathrm{C}_{2}$ & 1 & $\begin{array}{l}\mathrm{C}_{2} \times \mathrm{C}_{2} \\
\mathrm{~S}_{3} \\
\mathrm{~S}_{4}\end{array}$ & $\begin{array}{l}1 \\
1 \\
3\end{array}$ & $\begin{array}{l}S_{3} \\
D_{7}\end{array}$ & $\begin{array}{l}1 \\
1\end{array}$ & $\begin{array}{l}\mathrm{D}_{4} \\
\text { PGL }(2,16)\end{array}$ & $\begin{array}{l}1 \\
1\end{array}$ & $\begin{array}{l}\text { PGL }(2,16) \\
\text { PGO }(3,16)\end{array}$ & $\begin{array}{l}1 \\
1\end{array}$ & PGL $(2,16)$ & 1 \\
\hline
\end{tabular}


k (4-3) الأقواس k المختلفة بتأثير الاستقامة

الجدول رقم (4) يتضمن تصنيف الأقواس-k المختلفة بتأثير الاستقامة إذ أن Nk* يمثل عدد الأقواس

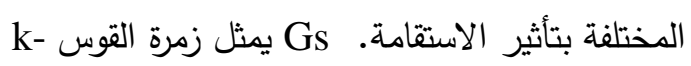

جدول رقم (9) - (9)

\begin{tabular}{|c|c|c|c|c|c|c|c|c|c|c|c|}
\hline \multicolumn{2}{|c|}{$\begin{array}{l}\mathrm{k}=5 \\
\mathrm{Nk}^{*}=1\end{array}$} & \multicolumn{2}{|c|}{$\begin{array}{l}\mathrm{k}=6 \\
\mathrm{Nk}^{*}=3\end{array}$} & \multicolumn{2}{|c|}{$\begin{array}{l}\mathrm{k}=7 \\
\mathrm{Nk}^{*}=2\end{array}$} & \multicolumn{2}{|l|}{$\begin{array}{l}\mathrm{k}=8 \\
\mathrm{Nk}^{*}=2\end{array}$} & \multicolumn{2}{|l|}{$\begin{array}{l}\mathrm{k}=9 \\
\mathrm{Nk}^{*}=2\end{array}$} & \multicolumn{2}{|l|}{$\begin{array}{l}\mathrm{k}=10 \\
\mathrm{Nk}^{*}=1\end{array}$} \\
\hline Gs & \# & Gs & \# & Gs & \# & Gs & \# & Gs & \# & Gs & \# \\
\hline $\mathrm{C}_{2} \times \mathrm{C}_{2}$ & 1 & $\begin{array}{l}\mathrm{C}_{2} \times \mathrm{C}_{2} \\
\mathrm{~S}_{3} \\
\mathrm{~S}_{4}\end{array}$ & $\begin{array}{l}1 \\
1 \\
1\end{array}$ & $\begin{array}{l}S_{3} \\
D_{7}\end{array}$ & $\begin{array}{l}1 \\
1\end{array}$ & $\begin{array}{l}\mathrm{D}_{4} \\
\text { PGL }(2,16)\end{array}$ & $\begin{array}{l}1 \\
1\end{array}$ & $\begin{array}{l}\text { PGL }(2,16) \\
\text { PGO }(3,16)\end{array}$ & $\begin{array}{l}1 \\
1\end{array}$ & PGL $(2,16)$ & 1 \\
\hline
\end{tabular}




\section{العصادر}

[1] Aziz, S.M., (2001), "On Lower Bound for Complete (k,n)-arc in PG(2,q)", M.Sc. Thesis, University of Mosul .

[2] Chao,J.M. and Kaneta. H., (2001), "Classial arcs in PG(r,q) for $23 \leq q \leq 29$ " Discrete Mathematics 226,p.p.377-385

[3] Coolsaet, K. and Sticker, H., "A full classification of complete k-arcs in PG(2,23) and PG(2,25)", Journal of Combinatorial Designs

[4] Coolsaet, K. and Sticker, H., (2009),"A full classification of complete k-arcs in PG(2,27)".

[5] Hasan, F. A. (2004), "On some complete arcs and Algebraic Curves," M. SC. Thesis, University of Mosul.

[6] Hirschfeld, J.W.P., (1979), "Projective Geometrics over Finite Fields", Oxford University Press, Oxford.

[7] Hirschfeld, J. W. P. and Storme, L., (2001), "The packing problem in statistics, coding theory and finite projective spaces : Update 2001", Submitted.

[8] Thomas, A.D. and Wood, G.V., (1980), "Group Tables", Shiva Publishing Ltd.

[9] Yasin, A.L., (1986), "Cubic arcs in the projective plane of order eight", Ph.D. Thesis, University of Sussex .

[10] Younis, H.,(1989), "Classification of k-arcs in the projectivety plane PG(2,16)", M.Sc. Thesis, University of Mosul. 